\title{
Oxytetracycline in the treatment of ocular rosacea: a double-blind trial
}

\author{
R. S. BARTHOLOMEW, ${ }^{1}$ B. J. REID,${ }^{2}$ M. J. CHEESBROUGH, ${ }^{3}$ \\ MARGARET MACDONALD,${ }^{4}$ AND N. R. GALLOWAY ${ }^{5}$
}

From the 'Department of Ophthalmology, University of Edinburgh; the ${ }^{2}$ Department of Dermatology, Royal Infirmary, Edinburgh; the ${ }^{3}$ Department of Dermatology, Royal Infirmary, Lindley, Huddersfield; the ${ }^{4}$ Department of Ophthalmology, Royal Infirmary, Edinburgh; and the ${ }^{5}$ Department of Ophthalmology, Nottingham General Hospital

SUMMARY Thirty-five patients with ocular rosacea were admitted to a trial of systemic oxytetracycline, $250 \mathrm{mg}$ b.d. for 6 weeks. Oxytetracycline produced a significantly higher number of remissions than the placebo, $11 / 35$. With repeated or continuous treatment $19 / 35$ patients achieved a sustained remission for 8 months. There were no side effects. The nonspecific signs of ocular rosacea responded well to the treatment, but no permanent change in the conjunctival or corneal vascularisation occurred.

Rosacea is a common condition affecting the skin of the face, with less frequent involvement of the eyes. Nonspecific ocular complications are similar to those found in patients with seborrhoea, blepharitis, recurrent chalazia, styes and trichiasis, hyperaemic conjunctivitis, with occasional marginal corneal infiltrates or ulcers. Specific complications include nodular conjunctivitis and episcleritis, which are rare, and the typical progressive keratitis which occurs in $5 \%$ of patients with rosacea. ${ }^{1}$

Oxytetracycline given orally in low dosage for more than 6 weeks is a well recognised treatment for rosacea of the $\operatorname{skin}^{2-4}$ and is also reassuringly safe..$^{5-7}$ Tetracycline has been used topically in ocular rosacea ${ }^{8}$ and there is increasing evidence that rosacea keratitis may also respond to systemic tetracyclines. ${ }^{19-11}$

The purpose of this study was to assess the validity of these observations by means of a double-blind limited cross-over trial.

\section{Material and methods}

Patients attending dermatology clinics with confirmed rosacea were questioned for possible eye symptoms. Sixty-five were subsequently screened by an ophthalmologist and were admitted to the trial if

Correspondence to Dr R. S. Bartholomew, Department of Ophthalmology, University of Edinburgh, Chalmers Street, Edinburgh EH3 9HA. their symptom and sign score (see below) reached a minimum level and if there were no general contraindications to the use of tetracyclines-pregnancy and lactation, hepatic or renal disease, drug allergy, or chronic bronchitis. Thirty-five patients were admitted to the trial.

Symptoms and signs were scored as absent (0), moderate (1), or severe (2). Symptoms were listed as: irritation, discharge, swelling of lids, redness, photophobia, pain, loss of sight. Signs were listed as: redness, swelling, discharge and chalazia for the eyelids, and redness, nodules, dilatation of vessels, tortuosity of vessels and microaneurysms for the conjunctiva and new vessels, superficial punctate keratitis, infiltrates, ulcers, and healed scars for the cornea. Left and right eyes were assessed separately. The maximum score for either eye was therefore 14 points for symptoms and 28 points for signs. A minimum of $25 \%$ score for symptoms and signs was required for admission to the trial.

On admission to the trial treatment was started with $250 \mathrm{mg}$ unmarked identical capsules containing either oxytetracycline dihydrate (Clinimycin) or a placebo of identical appearance composed of dextrose. Either capsule was taken in a dose of one capsule twice daily on an empty stomach. No local eye treatment was given.

The trial was divided into treatment periods. These were defined as a 6-week period on the drug or placebo followed by a 6-week observation period on 
no drug (to allow for possible carry-over effects). Patients were kept on the trial for a minimum of 3 treatment periods. They were examined every 6 weeks.

Those who responded to the first course of treatment were left untreated but received the same agent if a relapse occurred. Those who failed to respond to the initial treatment were crossed over to the other agent for the next period of treatment. Thus any patient who failed to respond to the initial treatment always received the active drug. Those patients responding to the initial agent were not crossed over in case possible delayed response affected treatments in a relapsing case. All those involved in the clinical examination of the patients were unaware whether drug or placebo was being administered. The trial was thus double-blind with limited cross-over.

Remissions and relapses were calculated according to the change in the symptoms and signs score. A full remission required a reduction of $>60 \%$ and a partial remission between 30 and $60 \%$. A relapse required an increase of $>30 \%$ on the score. A score $<30 \%$ above or below the previous score was deemed to be no change.

ASSESSMENT OF RESULTS

(a) The number of remissions (partial and full) was compared statistically ( $\chi^{2}$ test) with the number who did not remit-for the first treatment period.

(b) The subsequent periods of treatment were not randomly allocated and therefore not subject to statistical analysis. The results are listed according to the number of remissions obtained after one, 2 , or 3 courses of the drug or placebo, and whether such remissions were sustained or not after cessation of treatment.

(c) Side effects were noted.

(d) The response of the nonspecific and specific signs of ocular rosacea to treatment by oxytetracycline and placebo were noted.

\section{Results}

Details of the 35 patients admitted to the trial are listed in Table 1.

The remissions over the first treatment period are shown in Table 2 . There was a significantly higher rate of remission (partial and full) for patients taking oxytetracycline $(\mathrm{p}<0 \cdot 05)$.

Complications/side effects of the treatment. Two patients withdrew from the trial. One complained of nausea and sickness and refused to take any more tablets; the tablets were subsequently found to be the placebo. The other patient developed a corneal ulcer and had to be given specific topical treatment.
Table 1 Details of 35 patients in oxytetracycline trial

\begin{tabular}{lll}
\hline & $\begin{array}{l}\text { No. of } \\
\text { patients }\end{array}$ & $\begin{array}{l}\text { Time of } \\
\text { follow-up } \\
\text { (months) }\end{array}$ \\
\hline $\begin{array}{l}\text { (1) Remission maintained after a course } \\
\text { of drug }\end{array}$ & 5 & $3-6$ \\
$\begin{array}{l}\text { (2) Remission maintained after 2 courses } \\
\text { of drug }\end{array}$ & 7 & $2-5$ \\
(3) Remission maintained after 3 courses & & $2-5$ \\
of drug & 4 & $2-5$ \\
\hline $\begin{array}{l}\text { Sustained remissions after cessation of drug } \\
\text { (4) Remission maintained only by }\end{array}$ & 16 & \\
treatment & 3 & $5-8$ \\
(5) Remission maintained by placebo & 4 & $3-12$ \\
(6) Unchanged by treatment with drug & 2 \\
(7) Unchanged by treatment with placebo & 5 \\
(8) Made worse by drug & 2 \\
(9) Made worse by placebo & 1 \\
(10) Withdrawn from trial & 2 \\
\hline Total patients & 35 \\
\hline
\end{tabular}

No other systemic or local complications were attributable to the oxytetracycline or placebo.

Response of individual signs to treatment. The nonspecific signs-lid swelling, redness, blepharitis, chalazia, and conjunctival hyperaemia responded well to treatment. Conjunctival nodules, seen in one patient, regressed during treatment. Microaneurysms developed during exposure to oxytetracycline. Corneal infiltrates, marginal ulcers, and superficial punctate erosions responded to treatment. No change was noted in new vessels of the cornea or healed scars of the cornea.

There was a delay in response to oxytetracycline of about 2 weeks in most patients. Relapses generally occurred 2-3 weeks after stopping oxytetracycline but were occasionally immediate.

\section{Discussion}

It is difficult in ocular rosacea, where spontaneous exacerbations and remissions occur, to be sure whether clinical improvement is related to specific drug treatment or is merely part of the natural

Table 2 Remissions/nonremissions for oxytetracycline/placebo over first treatment period

\begin{tabular}{lccc}
\hline & Oxytetracycline & Placebo & Total \\
\hline No. of remissions & 11 & 5 & 16 \\
No. without remission & 6 & 13 & 19 \\
Total & 17 & 18 & 35 \\
\hline$x^{2}=4 \cdot 8, \mathrm{p}<0 \cdot 05$. & & &
\end{tabular}


variability of the disease. This trial showed a significantly greater incidence of remissions with oxytetracycline than with a placebo. Assessments were made after 6 weeks treatment with oxytetracycline $250 \mathrm{mg}$ b.d. On this dosage patients who responded began to do so about 2 weeks after starting treatment. Patients who relapsed after initial improvement did so about 2 weeks after stopping oxytetracycline. Repeated courses of treatment with the same dose achieved lasting remission 3-6 months after stopping treatment. Three patients required continuous treatment to maintain remission for periods of 5-8 months.

This regimen of treatment with oxytetracycline is that used successfully by dermatologists in the treatment of rosacea of the face. In their ocular trial Jenkins et al. " found poor results with tetracycline $250 \mathrm{mg}$ q.i.d. for 3 weeks followed by one week off treatment. They subsequently increased the dose to $250 \mathrm{mg}$ q.i.d. for 4 weeks. Maximum remission occurred 4 days to 3 weeks after starting treatment. Thirty-six of their 37 patients improved. Fifteen obtained complete remission. Five needed to be kept on oxytetracycline. These results are similar to ours, which also confirm the results of Brown and Shahinian. ${ }^{10}$

The remissions were mainly due to improvement in the nonspecific signs of rosacea-blepharoconjunctivitis. Acute signs-corneal infiltrates and nodules- -aiso improved, but there was no reduction in the vascular element of conjunctiva or cornea; indeed in one patient fresh microaneurysms appeared during a successful course of treatment.

Ocular rosacea responds to low-dosage treatment with oxytetracycline, $250 \mathrm{mg}$ b.d. Remissions may be obtained after one, 2, or 3 6-week courses or may require continued therapy. There is a 2 -week lag in response to initial treatment and a variable carry-over effect after stopping treatment.

Systemic oxytetracycline is thus a useful and safe treatment for ocular rosacea as well as rosacea of the face.

\section{CONCLUSIONS}

(1) Oxytetracycline in a dose of $250 \mathrm{mg}$ b.d. yielded more remissions than the placebo, $11 / 17(65 \%)$ over a period of 12 weeks ( 6 weeks on treatment, 6 weeks without treatment). (2) Oxytetracycline produced remissions in $19 / 35(54 \%)$ with repeated courses or continuous treatment. (3) Oxytetracycline sustained remissions after cessation of treatment in 16/35 $(45 \%)$. (4) No complications or side effects were attributable to oxytetracycline. (5) Nonspecific signs responded well to treatment by oxytetracycline. (6) No permanent change or improvement in conjunctival microaneurysms, corneal vascularisation, or scarring was seen.

\section{References}

1 Thygeson P. In: Sorsby A, ed. Systemic Ophthalmology. London: Butterworth, 1951.

2 Sneddon IB. A clinical trial of tetracycline in rosacea. $\mathrm{Br} \mathrm{J}$ Dermatol 1966; 78: 649-52.

3 Marks R, Ellis J. Comparative effectiveness of tetracycline and ampicillin in rosacea: a controlled trial. Lancet. 1971; ii: 1049-52.

4 Rook A, Wilkinson DS, Ebling FJG, eds. Textbook of Dermatology. 2nd ed. Oxford and Edinburgh: Blackwell, 1972.

5 Osment LS, Hammack WJ. The long-term use of tetracycline in acne vulgaris: effect on blood elements. $S$ Med J 1970; 63: $1156-8$.

6 Gilgor RS. Complications of tetracycline therapy for acne. $N C$ Med J 1972; 33: 331-3.

7 Delaney TJ, Leppard BJ. MacDonald DM. Effects of long term treatment with tetracycline. Acta Derm Venereol (Stockh) 1974; 54: 487-9.

8 Roper Hall MJ. Ocular aspects of rosacea. Trans Ophthalmol Soc UK 1966; 86: 727-32.

9 Marmion VJ. Tetracyclines in the treatment of ocular rosacea. Proc R Soc Med 1969; 62: 11-2.

10 Brown SI. Shahinian L. Diagnosis and treatment of ocular rosacea. Trans Am Acad Ophthalmol Otolaryngol 1977; 85: $779-86$.

11 Jenkins MS. Brown SI, Lempert SL, Weimber GRJ. Ocular Rosacea. Am J Ophthalmol 1979; 88: 618-22. 